Herz $2016 \cdot 41: 361$

DOI 10.1007/s00059-016-4449-x

Online publiziert: 13. Juni 2016

(c) Springer Medizin Verlag 2016

CrossMark

\author{
H. Thiele' $\cdot$ R. Dörr ${ }^{2}$ \\ ${ }^{1}$ Medizinische Klinik II (Kardiologie, Angiologie, Intensivmedizin) - Universitäres Herzzentrum Lübeck, \\ Universitätsklinikum Schleswig-Holstein (UKSH), Lübeck, Deutschland \\ ${ }^{2}$ Praxisklinik Herz und Gefäße, Dresden, Deutschland
}

\title{
Morphologie versus Funktion in der Ischämiediagnostik - die entscheidende Frage
}

hilfen werden durch Achenbach und Mitarbeiter elegant dargestellt.

Die Relevanz der Ischämielast, die mittels unterschiedlicher Verfahren bestimmt werden kann, im Vergleich zur Koronarmorphologie in Bezug auf die Notwendigkeit einer Revaskularisation aus prognostischer Sicht ist durch das Fehlen ausreichend großer prospektiver Studien derzeit schwierig, wie Hacker und Heber zeigen.

Die bisher größte randomisierte Vergleichsstudie zum Vergleich CT-Koronarangiographie versus funktionelle Ischämiediagnostik für die viel diskutierte Frage Morphologie oder Funktion wird durch das Team von Hoffmann et al., einem der Hauptautoren der PROMISEStudie, noch einmal vertieft dargestellt. Ebenso wird versucht, daraus mögliche Schlussfolgerungen für die klinische Praxis abzuleiten.

Sinnvoll erscheint natürlich die Kombination aus Anatomie und funktioneller Relevanz, wie es in der sogenannten Hybrid-Bildgebung, aber auch der anatomischen Beurteilung mittels CT-Koronarangiographie in Kombination mittels der CT-FFR erfolgt. Die aktuelle - zum jetzigen Zeitpunkt noch nicht ausreichende - Datenlage für eine breite klinische Anwendung wird in den beiden Artikeln von Kaufmann und Büchel als auch von Leber beleuchtet.

Die CT-Angiographie wird basierend auf der guten bisherigen Datenlage mit Sicherheit in Zukunft eine größere Rolle bei der Diagnostik der KHK spielen, wie Katus und Mitarbeiter darstellen. Wie sich das am besten in der Ausbildung und auch im klinischen Alltag für den Kardiologen auch aus Sicht der Deutschen Gesellschaft für Kardiologie implementieren lässt, ist eine wichtige Frage, da die Kompetenz für die Behandlung der KHK zweifelsohne in der Hand der Kardiologen liegt.

Unabhängig von aller Diskussion der Vorfelddiagnostik bleibt die invasive Koronarangiographie die Referenzmethode für die Diagnostik und auch die nachfolgende interventionelle Behandlung einer KHK. Es bedarf aber insbesondere in Deutschland des differenzierten Einsatzes der bildgebenden nichtinvasiven Verfahren und der gezielteren invasiven Diagnostik, damit wir nicht weiterhin der zweifelhafte Weltmeister der invasiven Diagnostik der stabilen KHK bleiben.

Wir hoffen, dass Ihnen unser Heft wieder gefällt und Sie viele Anregungen finden, die Sie in Ihrer täglichen Praxis übernehmen können.

Ihre

Holger Thiele und Rolf Dörr

\section{Korrespondenzadresse}

\section{Prof. Dr. med. H. Thiele}

Medizinische Klinik II (Kardiologie, Angiologie, Intensivmedizin) - Universitäres Herzzentrum Lübeck, Universitätsklinikum SchleswigHolstein (UKSH)

Ratzeburger Allee 160, 23538 Lübeck, Deutschland

holger.thiele@uksh.de

Interessenkonflikt. H. Thiele und R. Dörr geben an, dass kein Interessenkonflikt besteht. 